\title{
ALP-photon Mixing in Pulsar Magnetospheres
}

\author{
Avery Archer* \\ DePauw University \\ E-mail: averyarcherdepauw.edu \\ James Buckley \\ Washington University in St. Louis \\ E-mail: buck1eydwust1.edu
}

\begin{abstract}
Some extensions of the Standard Model suggest the existence of light, pseudo-scalar particles such as axions or axion-like particles (ALPs). Such particles couple to photons in the presence of an external magnetic field. As a result of this coupling ALP-photon oscillations will affect the propagation of very-high-energy gamma rays in strong magnetic field environments, as in the case of magnetosphere of pulsars. The emission regions of very-high-energy photons from pulsars are thought to be limited by the opacity of the magnetosphere due to the strong magnetic fields present. ALP-photon mixing in the inner pulsar magnetosphere allows for ALPs to propagate unimpeded by pair attenuation leading to an observable modification to the pulsar light-curve. We simulated the propagation of very-high-energy photons through a pulsar magnetosphere where ALP-photon mixing is present. For a range of ALP parameters and magnetic field strengths, ALP-photon mixing is shown to alter the opacity of pulsar magnetospheres to very-high-energy photons.
\end{abstract}

36th International Cosmic Ray Conference -ICRC2019-

July 24th - August 1st, 2019

Madison, WI, U.S.A.

${ }^{*}$ Speaker. 


\section{Introduction}

Axions are hypothetical light pseudoscalar particles that are predicted from the Peccei-Quinn solution to the strong-CP problem in QCD. These QCD axions are also a strong candidate for cold dark matter, with the correct density in the current universe (for a range of masses and coupling constants) and while quite light ( $\mu \mathrm{eV}$ to $\mathrm{meV}$ ) being non-relativistic at the time of galaxy formation. In addition to these QCD axions, other axion-like-particles (ALPs) appear in string theories with small extra dimensions resulting in a large number of axion fields (the so-called axiverse) that may play a role in explaining the dark matter or even dark energy. Axions or ALPs are pseudoscalers which have a two-photon coupling through an interaction term of the form:

$$
\mathscr{L}_{a \gamma \gamma}=-\frac{1}{4} g_{a \gamma} F_{\mu v} \tilde{F}^{\mu v} a=g_{a \gamma} \vec{E} \cdot \vec{B} a
$$

This so-called Primakoff effect is exploited in experiments like ADMX [1] (proposed by Sikivie [2]) that use large magnetic fields in a resonant cavity to detect the microwave cavity-mode photons created by this interaction. The QCD axion has a direct relation between its mass and the energy scale at which the Peccei-Quinn symmetry is violated. Furthermore, if the axions constitute the dark matter one can make robust predictions of the coupling constant $g_{a \gamma \gamma}$ as a function of mass (with a small dependence on the details of possible direct interactions of axions with fermions [3, $4,5,6])$. ALPs are not restricted to a direct mass-coupling relationship, expanding the parameter space for the ALP mass and photon coupling strength.

The presence of ALPs can play an important role in astrophysical processes and can yield observable effects. The two-photon coupling allows for ALP-photon oscillations in the presence of an external magnetic field. Several studies have been conducted investigating the effects of ALP-photon oscillations on the very-high energy (VHE; $E>100 \mathrm{GeV}$ ) spectra of distant blazars, see [7] for a recent review of limits. Pulsar magnetospheres can serve as another astrophysical environment for studying ALP-photon oscillations. Previous pulsar studies [8, 9, 10, 11, 12, 13] have been conducted investigating the effects of ALPs on pulsar observations at sub-GeV energies. Observations of pulsed emission from the Crab pulsar above $100 \mathrm{GeV}$ by VERITAS [14] and more recently up to $1 \mathrm{TeV}$ by MAGIC [15] motivate investigation of potential ALP signatures in the VHE band. More of the magnetosphere is opaque to the these VHE photons, limiting the potential regions for VHE emission. The existence of ALPs within certain parameters would decrease the opacity of the magnetosphere as ALP-photon oscillations occur in a similar fashion to those studied by observing high-redshift blazars. For typical parameters of pulsar magnetospheres, VHE photons fall within the strong mixing-regime for oscillations when the axion mass is $10^{-2}<m_{a}<10 \mu \mathrm{eV}$ and the axion-photon coupling constant is $10^{-10}<g<10^{-8} \mathrm{GeV}^{-1}$.

In this paper we focus on the VHE band to search for signatures of ALP-photon oscillations. To study the possible effects on observed light curves from pulsars we develop a simulation using a model pulsar magnetosphere using common theoretical models and introduce the effects of ALPphoton oscillations. 


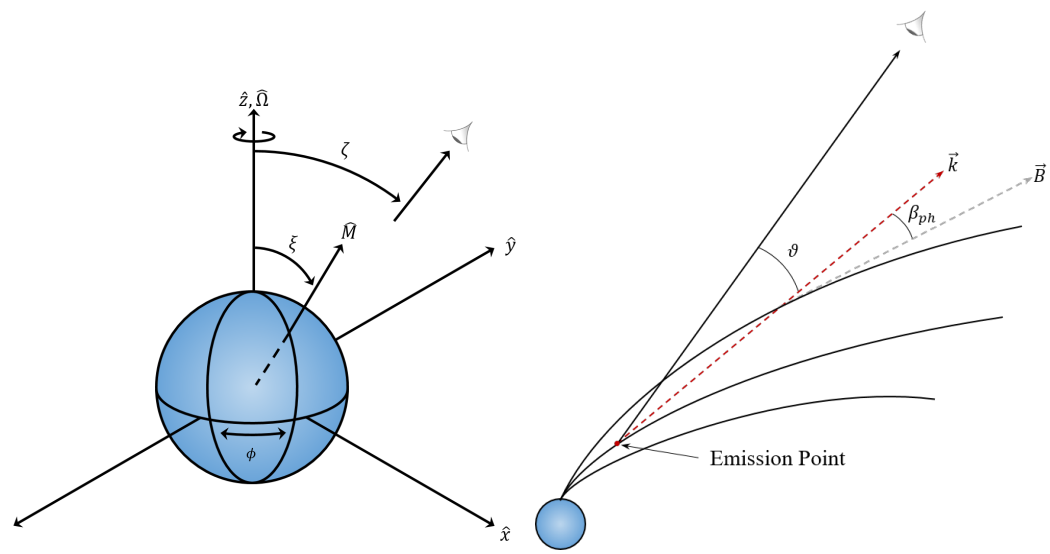

Figure 1: Left: Geometry used for pulsar simulations. The axis of rotation $\hat{\Omega}$ lies along the $z$-axis. Right: Schematic of the geometry used for calculation of pair production opacity. The emission point (red point) is identified in the diagram. The photon momentum vector $\vec{k}$ showing the direction of photon propagation is a dashed red arrow. Initially $\vec{k}$ is tangential to the field line but as the the photon propagates the angle $\beta_{p h}$ between $\vec{k}$ and $\vec{B}$ increases. $\vec{B}$ is shown by dashed gray arrow at one point along the photon's path. The line of sight to the observer is shown by the solid black arrow. An angle cut is applied for the angle $\vartheta$ to determine if a photon will be observed. The dipole magnetic field lines are shown as solid black lines.

\section{Simulating Photon Propagation and Pair Attenuation in Pulsar Magnetospheres}

\subsection{Pulsar Magnetosphere and Geometry}

This work adopts a simple standard model for pulsars, treating the magnetic field geometry to be given by the retarded potential vacuum dipole solution [16] and adopt the formalism used in [17]. The full geometry of the model used is depicted in Fig. W. The pulsar is centered at the origin and has a radius of $10 \mathrm{~km}$. The rotation axis $\hat{\Omega}$ lies along the $z$-axis. The angle of inclination of the magnetic moment $\xi$ is measured from the $z$-axis. The phase $\phi$ is defined to be zero when $\hat{M}$ is in the plane of the $+\hat{x}$-axis.

\subsection{Simulation of Photon Emission and Propagation}

An array of $n$ photons is generated for the simulation with some initial location $x_{i}$ in the magnetosphere and some energy $E$. We assume VHE photons are emitted through some relativistic process (e.g. curvature radiation or inverse Compton scattering), and thus are subject to relativistic beaming. The simulated photons have emission locations randomly distributed throughout common theoretical gap emission locations, the slot gap (SG) and outer gap (OG) regions, using Crab pulsar-like parameters: $M / R_{s}^{3}=10^{12} \mathrm{G}, P=0.033 \mathrm{~s}, R_{s}=10 \mathrm{~km}, \xi=\pi / 4, \zeta=8 \pi / 4$. Seed photons are given a narrow Gaussian distribution of energies at intervals throughout the VHE range.

It is assumed that emitted photons are beamed forward within a conical region centered along the magnetic field line at the photon's emission location. The angle between $\zeta$ and the magnetic field line along which a photon is generated is calculated. A selection cut is applied to this angle 


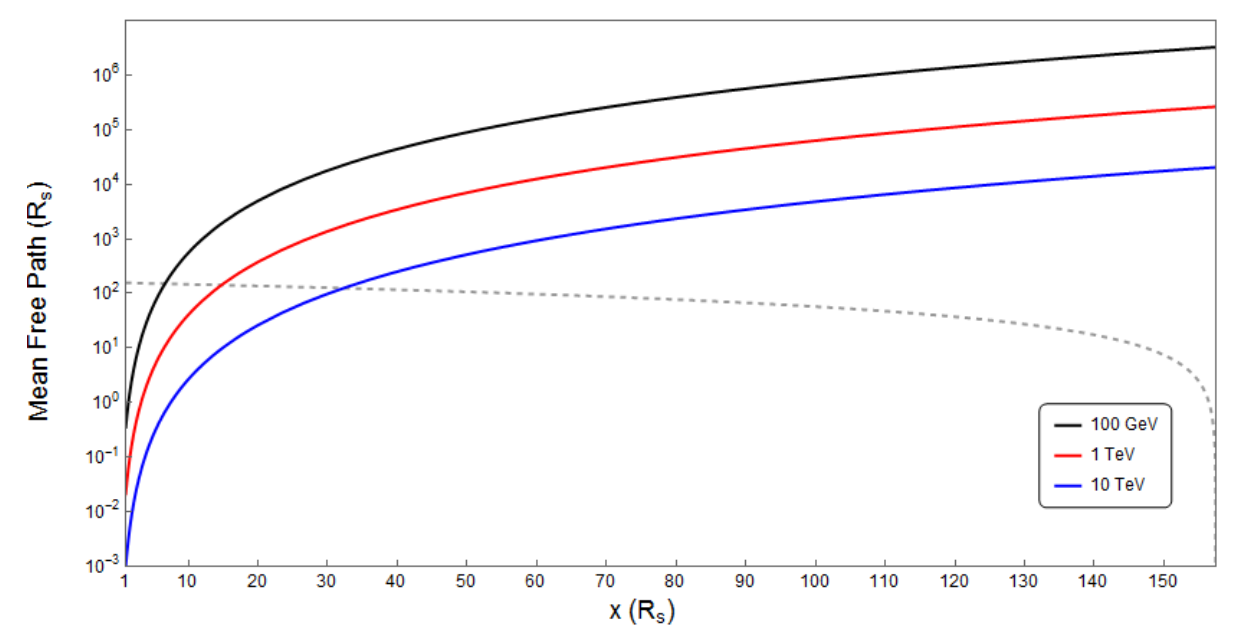

Figure 2: Plot of the $L$ of photons at various energies at any point from the stellar surface to the light cylinder. $L$ was calculated for a pulsar with inclination angle $\xi=\pi / 4$ and photons traveling along the $+\hat{x}$-direction. $L$ changes as a function of distance from the pulsar due to the falling magnetic field strength. The $x, y$ values are given in units of pulsar radii $\left(R_{S}\right)$. The dashed gray line is the remaining distance to the light cylinder from a given $x$-coordinate. Once the mean free path value exceeds the distance to the light cylinder photons are expected to survive propagation.

to account for beaming of the emission. If the angle is less than some value $\vartheta$ then the photon is "observed".

VHE photons traveling through a strong magnetic field interact with the field and may produce $e^{ \pm}$pairs. The attenuation of the VHE signal by photon-magnetic field interactions is considerable and therefore must be included to properly simulate photon propagation through the magnetosphere. We follow the formalism of [18] to calculate the pair production probability and the mean free path $L$ for simulated photons. This function for photon survival is highly dependent on the photon energy and the strength of the magnetic field component perpendicular to the photon propagation direction. Fig. $\square$ depicts $L$ for photons at different energies (100 GeV, $1 \mathrm{TeV}$ and $10 \mathrm{TeV})$ at different locations in the magnetosphere and shows that photons in the VHE band will easily pair produce if the photons are emitted near the stellar surface. The geometry used for the calculation of the probability of pair production for photons is shown in Fig. .

Photons that survive the $\vartheta$-cut are propagated through the magnetosphere. The path length is the distance between the photon emission point $r_{0}$ and the location at the light cylinder $R_{l c}$ reached along $\hat{k}$. This path is used to calculate the probability of survival. Light curves are created for the distribution of surviving photons and can be seen in Fig. [] and [1]. For comparison, light curves are also produced for the full initial distribution of photons assuming all survive showing the difference in peak strength and peak phase location when pair production is considered.

\section{ALP-photon Mixing}

As an initial step to gauge the efficacy of using pulsar magnetospheres to search for signatures of ALPs the relevant distance scales of pair production and ALP-photon oscillations are consid- 


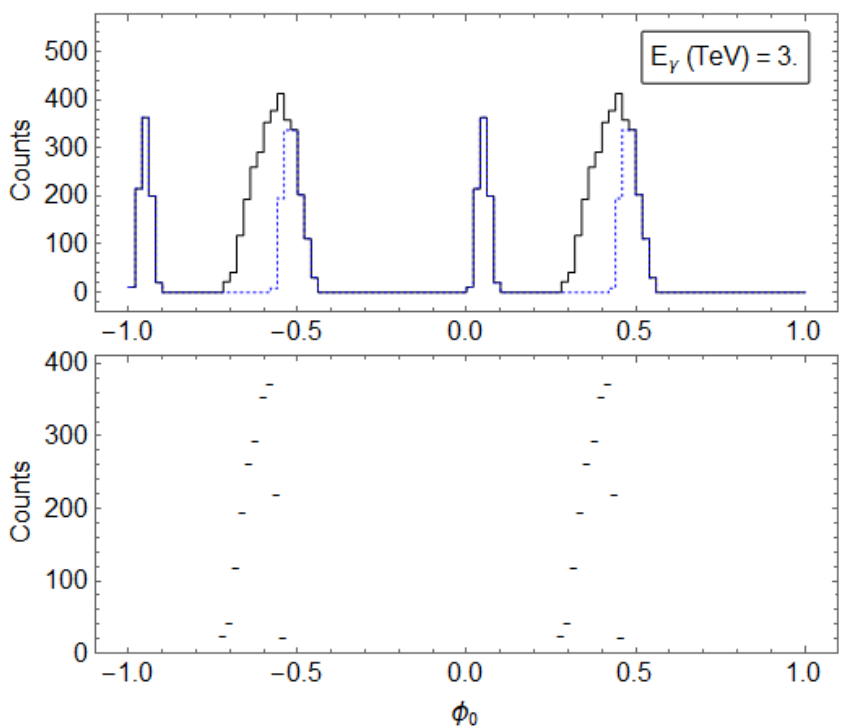

Figure 3: Light curve from simulations using Crab-like parameters and a discrete photon energy of $3 \mathrm{TeV}$ considering only geometric effects (black) and considering pair attenuation (blue). Residuals between the two curves are shown in the lower plot.

ered. If one physical process dominates for the energy range considered it is unlikely to find an observable effect on the propagation of VHE photons. The two processes need to be competitive for similar distance scales to expect an appreciable effect on the light curves and/or energy spectrum of pulsars. For pair production, $L$, serves as the relevant distance scale. The range of energies considered in our simulations and the associated range of $L$ are shown again in Figure $⿴$ in blue to compare directly with the relevant distance scale for ALP-photon oscillations. It has been shown [19] that for a simplified case of a photon traveling in a transverse magnetic field the probability of a single oscillation is given by

$$
P_{a \gamma}=\frac{1}{1+\left(E_{\text {crit }} / E_{\gamma}\right)^{2}} \sin ^{2}\left[\frac{B d g_{a \gamma}}{2} \sqrt{1+\left(\frac{E_{\text {crit }}}{E_{\gamma}}\right)^{2}}\right]
$$

where $E_{\text {crit }}$ is the energy threshold above which oscillation probability is maximal and independent of $E_{\gamma}$. From 3.$]$ it is clear the probability of conversion is maximal at some path distance $d=d_{\max }$ where $d_{\max }=\pi /(B g)$ for a given magnetic field strength and coupling constant. This quantity $d_{\max }$ becomes the relevant length scale to consider and is calculated using ALP-photon coupling constant values $g=10^{-8} \mathrm{GeV}^{-1}$ to $g=10^{-7} \mathrm{GeV}^{-11}$ over the range of values of $B$ encountered throughout

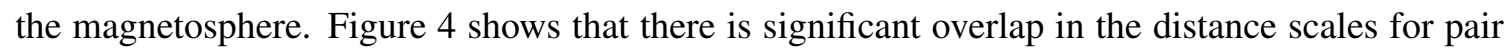
production and ALP-photon oscillations throughout the entire magnetosphere.

In the following we adopt the formalism described by [20]. The attenuation of VHE photons in pulsar magnetospheres due to pair production is understood to play a large role in the observed light curves of pulsars. Due to the strength of the magnetic field and the very high energy of photons considered in this work, pair production can not be excluded when formulating the mixing matrix. The addition of pair production effects to the ALP-photon mixing calculation comes in the form 


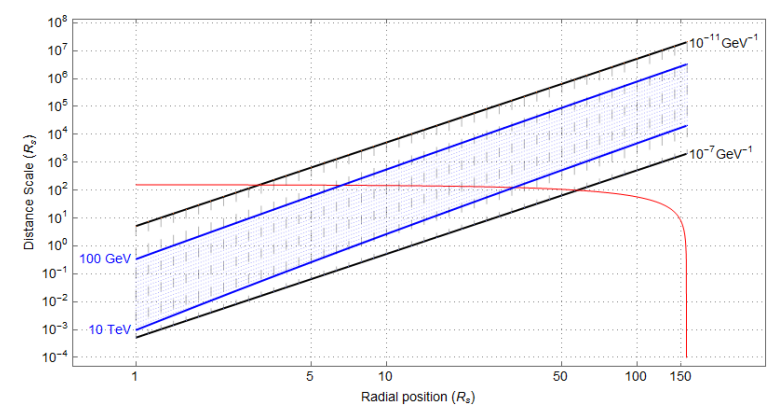

Figure 4: Comparison of relevant distance scales for pair-production and ALP oscillation probability for a pulsar with $\xi=\pi / 4, \zeta=\pi / 2$. The horizontal axis is the emission location of photons in units of stellar radii $\left(R_{S}\right)$ and goes from the stellar surface out to the light cylinder radius. The vertical axis is the distance scale for the two considered physical processes in units of stellar radii $\left(R_{S}\right)$. The mean free path for the range of energies considered $(100 \mathrm{GeV}$ to $10 \mathrm{TeV})$ is bounded by blue lines and shaded. The distance scale that maximizes ALP oscillation probability $(d=\pi /(B g))$ for the relevant range of coupling constant, $g,\left(10^{-11}\right.$ to $\left.10^{-7}\right)$ is bounded by black lines and hashed. The distance from the radial position to the light cylinder $R_{l c}$ is shown in red to indicate what distance scales are of interest for pulsar magnetospheres. From this figure it can be seen that there is a range of photon energies and coupling constants for which the distance scale relevant to pair production and ALP oscillation probability are comparable. This range lies naturally in the VHE regime for photon energies.

of an imaginary absorption term that is added to the plasma term of the mixing matrix $\mathscr{M}$ so that $\Delta_{p l} \rightarrow \Delta_{p l}+i /(2 L)$, where $L$ is the mean free path of pair production. The mixing matrix then takes the form

$$
\mathscr{M}=\left(\begin{array}{ccc}
\Delta_{p l}+\frac{i}{2 L} & 0 & \Delta_{a \gamma} \sin \psi \\
0 & \Delta_{p l}+\frac{i}{2 L} & \Delta_{a \gamma} \cos \psi \\
\Delta_{a \gamma} \sin \psi & \Delta_{a \gamma} \cos \psi & \Delta_{a}
\end{array}\right)
$$

where $\Delta_{p l}=-\omega_{p l}^{2} /\left(2 E_{\gamma}\right), \Delta_{a \gamma} \equiv B g / 2$, and $\Delta_{a}=-m_{a}^{2} /\left(2 E_{\gamma}\right)$. There is an additional term on the diagonal, $\Delta_{\mathrm{QED}}$ which comes from QED vacuum polarization but is excluded here. For the case of a pulsar magnetosphere this term is only necessary for photons emitted at or very near the stellar surface. Such cases are not considered in this study.

The probability that a photon beam initially in some state $\rho_{i}$ will be found in the $\rho_{f}$ state after propagating some distance $d$ is

$$
P_{\rho_{i} \rightarrow \rho_{f}}=\operatorname{Tr}\left[\rho_{\mathrm{f}} \mathscr{U} \rho_{\mathrm{i}} \mathscr{U}^{\dagger}\right]
$$

The density matrix for an unpolarized beam $\rho_{\text {unpol }}$ and the density matrix for an ALP state $\rho_{a}$ are

$$
\rho_{\text {unpol }}=\frac{1}{2}\left(\begin{array}{lll}
1 & 0 & 0 \\
0 & 1 & 0 \\
0 & 0 & 0
\end{array}\right) \quad \rho_{a}=\left(\begin{array}{lll}
0 & 0 & 0 \\
0 & 0 & 0 \\
0 & 0 & 1
\end{array}\right)
$$

We are interested in the case of an unpolarized beam of VHE photons propagating through a pulsar magnetosphere and the rate at which such photons convert to ALPs. The probability of 

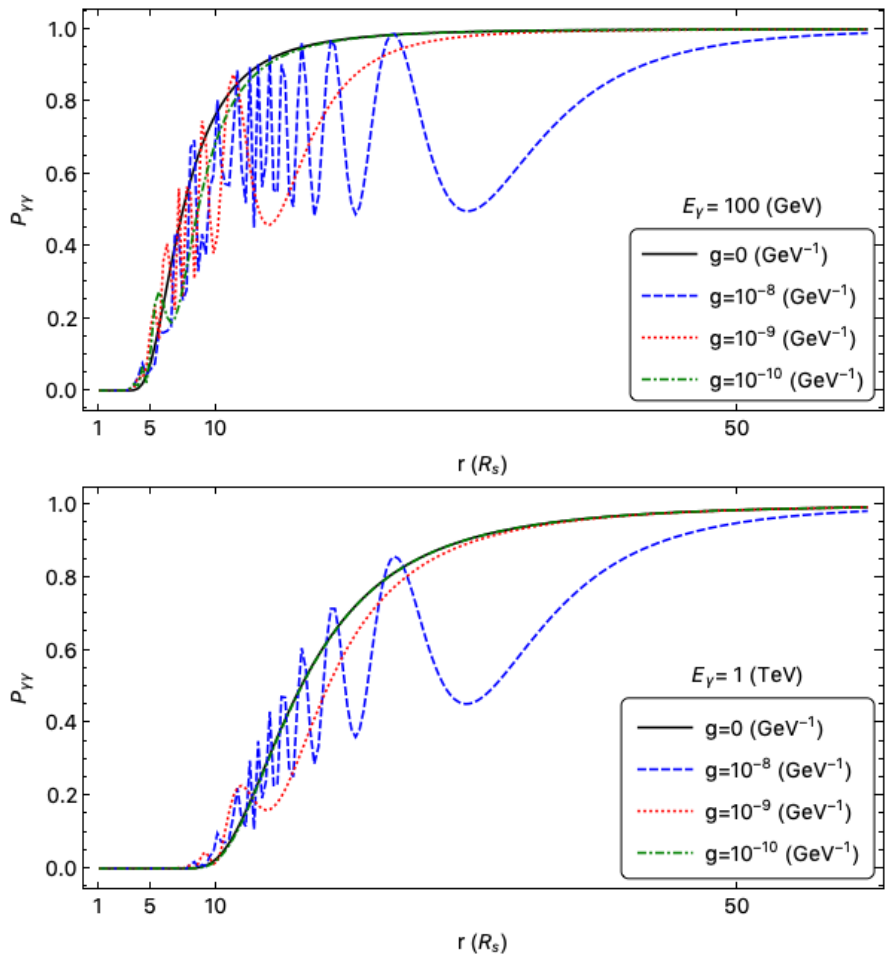

Figure 5: The probability of a photon emitted at positions throughout the magnetosphere remaining in a photon state as it reaches the light cylinder. $P_{\gamma \gamma}$ is calculated using the simplifying assumption that photons are traveling along the $\hat{x}$-axis. Top: $P_{\gamma \gamma}$ for $100 \mathrm{GeV}$ photons. Bottom: $P_{\gamma \gamma}$ for 1 $\mathrm{TeV}$ photons.

ALP-photon oscillations is given by

$$
P_{\gamma \rightarrow a}=\operatorname{Tr}\left[\rho_{\mathrm{a}} \mathscr{U} \rho_{\gamma} \mathscr{U}^{\dagger}\right]
$$

The probability of ALP-photon oscillation is calculated for positions throughout a pulsar magnetosphere for a photon beam with energy $E_{\gamma}=100 \mathrm{GeV}, 1 \mathrm{TeV}$ and $10 \mathrm{TeV}$, with the ALP-photon coupling taking a range of values. Figure $\square$ shows the probability that a photon emitted will remain in a photon state after propagating through the magnetosphere, the case of no ALP-photon coupling $(g=0)$ as a reference. The photon probability survival, $P_{\gamma \gamma}$, oscillates with distance from the stellar surface. For certain values of $g$ at certain energies $P_{\gamma \gamma}$ differs by as much as 0.5 from the the case where $g=0$ in the region from $10 R_{s}$ to $50 R_{s}$. At some points in the magnetosphere it can be seen that $P_{\gamma \gamma}$ is enhanced by ALP-photon mixing, while in other regions $P_{\gamma \gamma}$ is diminished. These regions shift with energy.

The recent observations by VERITAS and MAGIC of the Crab pulsar up to $>400 \mathrm{GeV}$ energies, paired with the natural overlap in $d_{\max }$ and $L$ at these energies motivates a more careful examination of ALP-photon oscillations on observable quantities like the light curves of the VHE emission. To study this effect, work is ongoing for a Monte Carlo simulation of the emission and propagation of VHE photons is performed using methods described in 2 . The parameters for each simulated photon (initial position, energy, phase position, path to observer) are used with Equation 
3.4 to calculate the probability of ALP-photon oscillation and the probability of photons surviving in either polarization state. The path of the photon is divided into small segments and the probability of survival is calculated for each segment to account for the change in magnetic field orientation due to the pulsar's rotation and magnetic field geometry at greater altitudes from the stellar surface. Rather than directly using the probability in a Monte Carlo calculation to accept or reject individual events, the probability of photon survival $P_{\gamma \gamma}$ for an individual photon position and energy is used as a weighting factor for the produced light curve and differential energy spectrum to preserve computational resources. The simulation and analysis are not fully completed and results in the form of lightcurves are forthcoming.

The 2011 VERITAS observations of VHE pulsed emission from the Crab and the following 2015 MAGIC observations of $\mathrm{TeV}$ pulsed emission from the Crab came as somewhat of a surprise because VHE photons are expected to be heavily attenuated in the strong magnetic fields of pulsar magnetospheres. Pair attenuation is expected to primarily affect the spectral index due the energy dependence of attenuation. The precise nature of the VHE emission is not yet understood or agreed upon, but current models do not allow for such high-energy emission near the stellar surface. ALPphoton mixing may allow for near-stellar-surface emission affecting the spectral index.

With new generation experiments such as Cherenkov Telescope Array, which will have a greater sensitivity to gamma rays than current generation telescopes, more VHE pulsars may be added to the catalog. With a population of VHE pulsars the ALP parameter space can be probed using the methods described in this work and constraints can be placed on the ALP mass and coupling constant. These constraints will complement the constraints from other astrophysical studies and experimental searches.

\section{References}

[1] T. M. Shokair et al. "Future directions in the microwave cavity search for dark matter axions". In: International Journal of Modern Physics A 29, 1443004 (July 2014), p. 1443004. DOI: $10.1142 /$ S0217751X14430040. arXiv: 1405.3685.

[2] P. Sikivie. "Experimental tests of the 'invisible' axion". In: Physical Review Letters 51 (Oct. 1983), pp. 1415-1417. DOI: 10.1103/PhysRevLett.51.1415.

[3] Jihn E. Kim. "Weak-Interaction Singlet and Strong CP Invariance". In: Phys. Rev. Lett. 43 (2 July 1979), pp. 103-107. DOI: 10.1103 / PhysRevLett . 43.103 . URL: https : //link.aps.org/doi/10.1103/PhysRevLett.43.103.

[4] M.A. Shifman, A.I. Vainshtein, and V.I. Zakharov. "Can confinement ensure natural CP invariance of strong interactions?” In: Nuclear Physics B 166.3 (1980), pp. 493-506. ISSN: 0550-3213. DOI: https://doi.org/10.1016/0550-3213(80)90209-6.

[5] M. Dine, W. Fischler, and M. Srednicki. "A simple solution to the strong CP problem with a harmless axion". In: Physics Letters B 104 (Aug. 1981), pp. 199-202. DOI: $10.1016 /$ $0370-2693$ (81) 90590-6.

[6] A. R. Zhitnitsky. "On Possible Suppression of the Axion Hadron Interactions. (In Russian)". In: Sov. J. Nucl. Phys. 31 (1980), p. 260. 
[7] M. Meyer and for the Fermi-LAT Collaboration. "Searches for Axionlike Particles Using Gamma-Ray Observations”. In: ArXiv e-prints (Nov. 2016). arXiv: 1611.07784.

[8] R. Jimenez, C. Peña-Garay, and L. Verde. "Is it possible to explore Peccei-Quinn axions from frequency-dependence radiation dimming?" In: Physics Letters B 703 (Sept. 2011), pp. 232-236. DoI: 10.1016/j.phys letb.2011.08.019. arXiv: 1102.1728.

[9] D. Chelouche et al. "Spectral Signatures of Photon-Particle Oscillations from Celestial Objects”. In: The Astrophysical Journal Supplement 180 (Jan. 2009), pp. 1-29. DOI: 10 . 1088/0067-0049/180/1/1. arXiv: 0806.0411.

[10] D. Lai and J. Heyl. "Probing axions with radiation from magnetic stars". In: Physical Review D 74.12, 123003 (Dec. 2006), p. 123003. DOI: 10.1103 / PhysRevD . 74.123003. eprint: astro-ph/0609775.

[11] R. Perna et al. "Signatures of Photon-Axion Conversion in the Thermal Spectra and Polarization of Neutron Stars". In: The Astrophysical Journal 748, 116 (Apr. 2012), p. 116. DOI: 10.1088/0004-637X/748/2/116. arXiv: 1201.5390 [astro-ph.HE] .

[12] Naoki Iwamoto. “Axion Emission From Neutron Stars”. In: Physical Review Letters 53 (12 Sept. 1984), pp. 1198-1201. DOI: 10.1103/PhysRevLett . 53.1198. URL: http: //link.aps.org/doi/10.1103/PhysRevLett.53.1198.

[13] B. Berenji, J. Gaskins, and M. Meyer. "Constraints on axions and axionlike particles from Fermi Large Area Telescope observations of neutron stars". In: Physical Review D 93 (4 Feb. 2016), p. 045019. DOI: 10.1103 / PhysRevD . 93 . 045019 . URL: http : / / link.aps.org/doi/10.1103/PhysRevD.93.045019.

[14] VERITAS Collaboration et al. "Detection of Pulsed Gamma Rays Above $100 \mathrm{GeV}$ from the Crab Pulsar". In: Science 334 (Oct. 2011), p. 69. DoI: 10.1126 / science. 1208192. arXiv: 1108.3797 [astro-ph.HE] .

[15] S. Ansoldi et al. "Teraelectronvolt pulsed emission from the Crab Pulsar detected by MAGIC". In: Astronomy and Astrophysics 585, A133 (Jan. 2016), A133. DOI: 10 . 1051 / 0004 6361/201526853. arXiv: 1510.07048 [astro-ph.HE] .

[16] A. J. Deutsch. "The electromagnetic field of an idealized star in rigid rotation in vacuo". In: Annales d'Astrophysique 18 (Jan. 1955), p. 1.

[17] F. C. Michel and H. Li. "Electrodynamics of neutron stars". In: Physics Reports 318 (Sept. 1999), pp. 227-297. DOI: 10.1016/S0370-1573(99)00002-2.

[18] V. S. Beskin, A. V. Gurevich, and Y. N. Istomin. Physics of the pulsar magnetosphere. Jan. 1993.

[19] G. G. Raffelt. Stars as laboratories for fundamental physics : the astrophysics of neutrinos, axions, and other weakly interacting particles. 1996.

[20] A. de Angelis, G. Galanti, and M. Roncadelli. "Relevance of axionlike particles for veryhigh-energy astrophysics". In: Physical Review D 84.10, 105030 (Nov. 2011), p. 105030. DOI: 10.1103 /PhysRevD .84.105030. arXiv: 1106.1132 [astro-ph.HE] . 